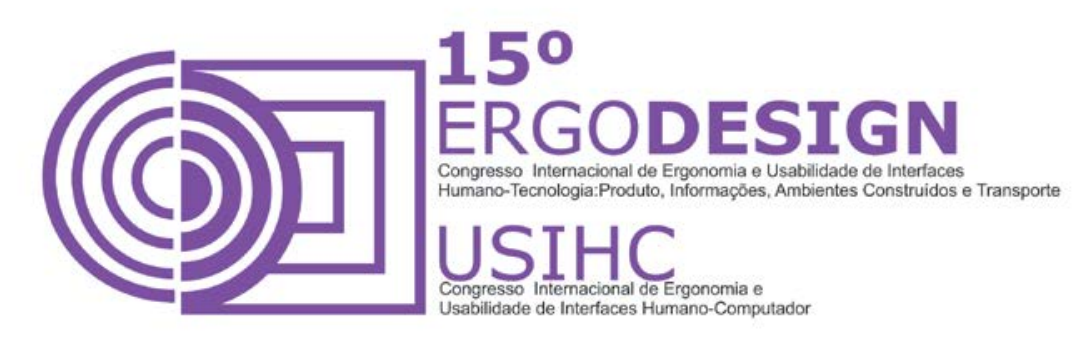

\title{
PARÂMETROS E REQUISITOS PARA O PROJETO DE ARTEFATOS PARA A ATIVIDADE DE DESENHO DA CRIANÇA COM PARALISIA CEREBRAL
}

\section{PARAMETERS AND REQUISITES FOR DEVICES DESIGN FOR DRAWING OF THE CHILD WITH CEREBRAL PALSY}

\author{
MARCELINO, Juliana Fonsêca de Queiroz (1); \\ MARTINS, Laura Bezerra (2)
}

(1) Universidade Federal de Pernambuco (UFPE), Mestra em Psicologia Clínica, Doutoranda em Design, professora assistente do Departamento de Terapia

Ocupacional da UFPE.

E-mail: jullifons@yahoo.com.br

(2) Universidade Federal de Pernambuco, Doutora em Arquiteratura, Professora Associada do Departamento de Design da UFPE

E-mail: bmatins.laura@gmail.com

\begin{abstract}
RESUMO
A criança com paralisia cerebral $(\mathrm{PC})$ atetoide apresenta dificuldade na programação e execução adequada dos movimentos voluntários, na coordenação de movimentos automáticos e na manutenção da postura, o que dificulta seu desempenho na atividade de desenho. Esta pesquisa trata-se de uma revisão de literatura realizada dentro do tema da pesquisa do doutorado em design que teve como objetivo estudar artefatos desenvolvidos para auxiliar o desenho da criança com PC e identificar a apresentação de parâmetros e requisitos no projeto destes artefatos. Os artigos analisados abordavam tanto artefatos digitais quanto físicos; os requisitos foram apresentados com maiores detalhes nos projetos de artefatos digitais.
\end{abstract}

Palavras-chave: paralisia cerebral, desenho, artefatos, design.

\section{ABSTRACT}

The child with cerebral palsy (CP) athetoid present difficulties in planning and proper execution of voluntary movements, coordination of automatic movements and to maintain posture, which hinders their performance on the drawing activity. This research it is a literature review conducted in the doctoral research topic in design aimed to study artifacts developed to aid the drawing of children with $\mathrm{CP}$ and identify the presentation parameters and requisites in the design of these artifacts. The 


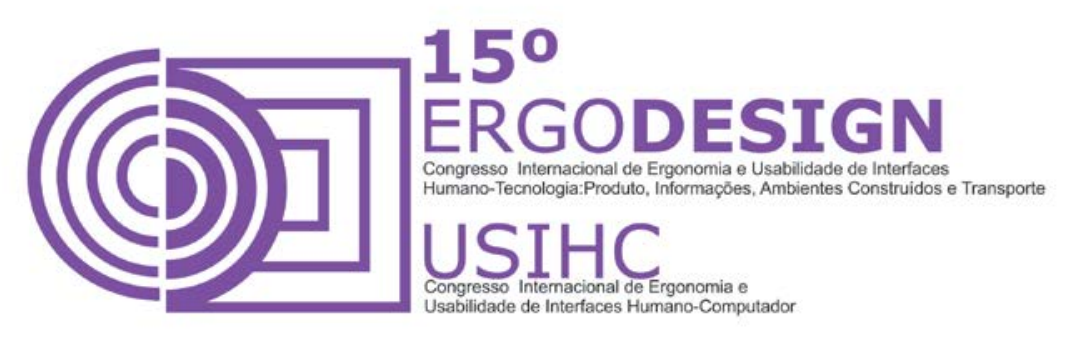

articles analyzed addressed both digital and physical artifacts; the requirements were presented in more detail in digital artifacts projects.

Keywords : cerebral palsy, drawing, artifacts, design.

\section{INTRODUÇÃO}

O problema que origina a exploração do tema deste trabalho é a dificuldade, ou mesmo impossibilidade, que crianças com sequelas de paralisia cerebral têm de desempenhar a atividade do grafismo, de desenhar, tão importante ao desenvolvimento das mesmas. Compreende-se que o design é uma área que pode contribuir na solução deste problema, uma vez que se faz necessário avaliar e intervir sobre o ambiente e os artefatos, bem como sobre outras variáveis, para obtenção de melhores resultados na usabilidade.

A Paralisia Cerebral (PC) refere-se a uma série heterogênea de síndromes clínicas, de etiologia não progressiva, que ocorre em um cérebro imaturo, caracterizada por distúrbios motores e alterações posturais permanentes, podendo ou não estar associada a alterações cognitivas (GAUZZI; FONSECA, 2004). É classificada de acordo com o tipo e a localização da alteração motora em: espástica (hemiplégica, diplégica, quadriplégica), discinética, atáxica, hipotônica e mista.

A PC discinética (PCDi) também denominada atetoide por Krigger (2006), caracteriza-se por coordenação motora ineficiente e alterações na regulação do tônus muscular. Apresenta uma dificuldade na programação e execução adequada dos movimentos voluntários, na coordenação de movimentos automáticos e na manutenção da postura. Esta forma corresponde a 8 a 15\% dos casos (GAUZZI; FONSECA, 2004). Este quadro interfere negativamente na possibilidade da criança de controlar seus movimentos.

Para Shumway-Cook e Woollacott (2003, p. 41), o controle do movimento é obtido pelo esforço cooperativo de muitas estruturas cerebrais, nos sistemas de percepção e ação. Ele não é tarefa simples, e está diretamente relacionado à função: "é uma atividade complexa de todo o organismo, direcionada ao desempenho de uma tarefa comportamental".

Assim, compreende-se que a dificuldade no controle dos movimentos apresentada pela criança com PCDi interfere negativamente no processo de grafismo, visto que é a precisão do movimento da criança sobre a superfície que vai determinar o desenho que ela tem a intenção de fazer. E, para isso, ela precisa manipular objetos, tais como lápis e giz de cera, ou seu próprio dedo ou objetos de interface digital. Sobre isso, Cazeiro e Lomônaco (2011) apontam a dificuldade da criança com PC em manipular objetos.

Conforme Meredieu (1993 apud CASSIS; FRANCISQUETTI, 2006), a elaboração do sistema gráfico é paralela à evolução psicomotora e ocorre de modo progressivo e evolutivo, por etapas. A partir do exposto, compreende-se que, a criança com sequela de paralisia cerebral, que tem comprometimento no desenvolvimento psicomotor, consequentemente, terá alteração no desenvolvimento das produções gráficas também.

Faz-se necessário conhecer a importância do desenho para o desenvolvimento infantil para se compreender que a disfunção hora discutida de fato é um problema, pois o grafismo é meio e fim. Meio no sentido de ser mais uma atividade que propicia estímulos para o 


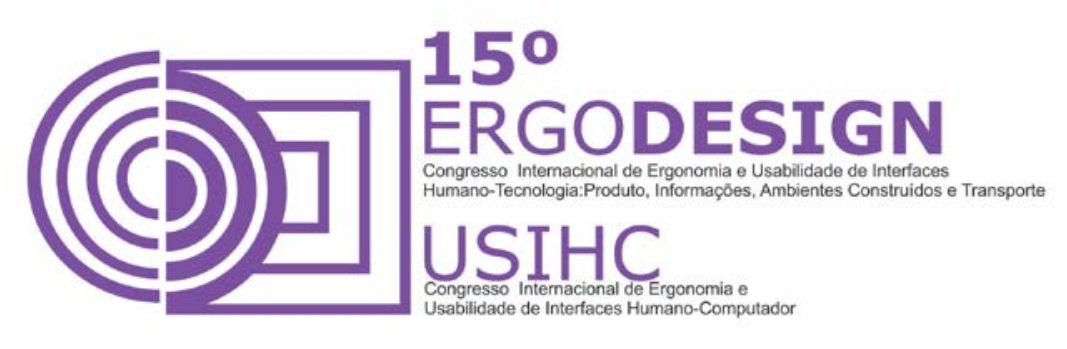

desenvolvimento da criança e fim no sentido de ser uma tarefa esperada a ser desempenhada dentro da sua faixa etária.

Segundo Cassis e Francisquetti (2006), o desenho é uma produção simbólica, uma expressão pessoal da criança de fragmentos do real que the foram significativos. Por meio do desenho, ela registra seu gesto em alguma superfície, e, inicialmente, o faz com qualquer instrumento que sirva de prolongamento para o corpo. Quanto a estes instrumentos, Cavaton e Barbato (2010) citam que lápis, giz de cera e papeis são meios culturais, disponibilizados pelas famílias e professores, com os quais a criança pequena desenha.

No início a criança produz marcas inicias ainda aleatórias, sem intenção de representar, que são as garatujas, e por estar em contato com inúmeras imagens desde seu nascimento, depois seu traçado passa a ser elaborado enquanto objeto semiótico. A possibilidade da criança de inventar e de produzir suas produções gráficas livres se relaciona com 0 desenvolvimento de sua capacidade criadora, com sua imaginação e fantasia (CAVATON; BARBATO, 2010). E ainda, Silva e Vasconcelos (2013) indicam que a experiência do desenho é importante para a constituição do sujeito.

Vários estudos têm relatado a relação entre a postura de pega do lápis e as habilidades de caligrafia. Sabe-se que existem artefatos projetados para facilitar essa pega, mas ainda pouco funcionais para a criança com PC atetoide (KAVAK; BUMIN, 2009). E nesta perspectiva de função é importante definir "usabilidade", que é a medida na qual um produto pode ser usado por usuários específicos para alcançar objetivos específicos com eficácia, eficiência e satisfação em um contexto específico de uso (NBR 9241-11, p.3).

Este estudo está sendo desenvolvido dentro da pesquisa do doutorado em design, na qual se pretende estabelecer parâmetros para o projeto de artefatos para a atividade de grafismo de crianças com paralisia cerebral do tipo atetoide. O objetivo deste estudo, porém, foi estudar artefatos desenvolvidos para auxiliar o desenho de crianças com PC e identificar a apresentação de parâmetros e requisitos no projeto destes artefatos. Entendendo-se parâmetro como "elemento importante a levar em conta, para avaliar uma situação ou compreender um fenômeno em detalhe" e requisito como "condição que se deve satisfazer para alcançar certo fim" (DIClO, 2015).

Os artigos analisados abordavam tanto artefatos digitais quanto físicos; os parâmetros e requisitos foram apresentados com maiores detalhes nos projetos de artefatos digitais. Como metodologia, foi realizada uma revisão de literatura nos idiomas português e inglês, com os seguintes descritores: "paralisia cerebral atetoide" / "cerebral palsy athetoid" combinados com "desenho"/ "drawing"; "escrita/ "writing"; "grafismo" e adaptação para lápis.

\section{RESULTADOS E DISCUSSÃO}

Neste momento inicial de levantamento de dados, que deu origem a este estudo, foram utilizados dois canais de busca: portal de periódicos capes e google scholar. Porém, alguns artigos foram encontrados no google scholar, mas a versão completa foi obtida no portal capes. E alguns outros foram encontrados no portal capes em língua japonesa e encontrados no google scholar na versão em inglês.

A seleção inicial dos artigos encontrados foi determinada pelo critério de relação com o tema da pesquisa, citado anteriormente. Especialmente no google scholar, a maioria das obras encontradas na busca foram excluídas por tratarem de uso do computador na atividade de 


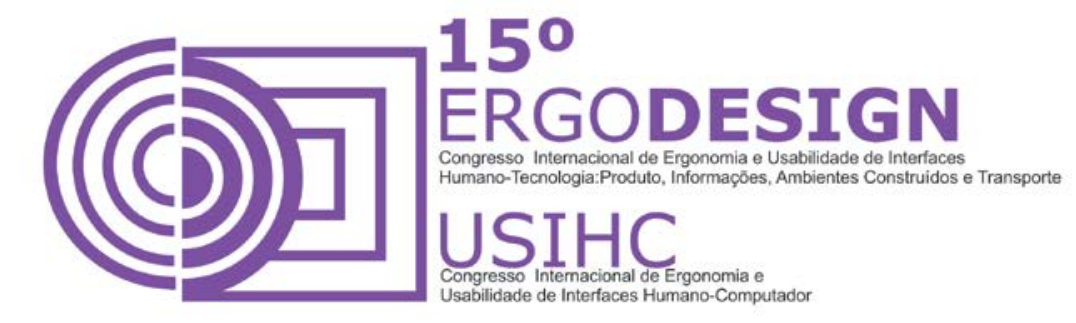

digitação, comunicação alternativa e outras patologias, ou paralisia cerebral sem relação alguma com a atividade de grafismo. Foram selecionados 6 artigos para este estudo (tabela 1), sendo 4 em inglês e 2 em português. Do total, 5 utilizaram recurso digital e apenas um indicou utilizar material de baixo custo.

Tabela 1

\section{ARTIGOS CIENTÍFICOS}

\begin{tabular}{|c|c|c|c|}
\hline TÍTULO & AUTORES & $\begin{array}{c}\text { ANO DE } \\
\text { PUBLICAÇÃO }\end{array}$ & PERIÓDICO \\
\hline $\begin{array}{l}\text { Movement-Accuracy Control in } \\
\text { Tetraparetic Cerebral Palsy : Effects } \\
\text { of Removing Visual Information of the } \\
\text { Moving Limb }\end{array}$ & $\begin{array}{l}\text { ROON, D.V.; } \\
\text { STEENBERGEN, } \\
\text { B.; } \\
\text { MEULENBROEK, } \\
\text { R.G. J. }\end{array}$ & 2005 & Motor Control \\
\hline $\begin{array}{l}\text { Development of drawing assist } \\
\text { system for patients with cerebral } \\
\text { palsy of the tension athetosis type }\end{array}$ & AOYAMA, $H$. et $A L$ & 2011 & $\begin{array}{l}\text { IEEE } \\
\text { International } \\
\text { Conference on } \\
\text { Robotics and } \\
\text { Automation }\end{array}$ \\
\hline $\begin{array}{l}\text { Assistive drawing device design for } \\
\text { Cerebral Palsy children }\end{array}$ & WU, F. et AL & 2013 & $\begin{array}{l}\text { Technology and } \\
\text { Disability }\end{array}$ \\
\hline $\begin{array}{l}\text { Drawing Assist System Considering } \\
\text { Nonperiodic Involuntary Movements }\end{array}$ & $\begin{array}{l}\text { NAKAO, T.; } \\
\text { SAKAMOTO, R.; } \\
\text { YANO, K. }\end{array}$ & 2013 & $\begin{array}{l}\text { Symposium on } \\
\text { analysis, design } \\
\text { and evaluation } \\
\text { of human- } \\
\text { machine } \\
\text { systems }\end{array}$ \\
\hline $\begin{array}{l}\text { Utilização de dispositivos assistivos } \\
\text { por alunos com deficiência em } \\
\text { escolas públicas }\end{array}$ & $\begin{array}{l}\text { PLOTEGHER, C. } \\
\text { B.; } \\
\text { EMMEL, M.L.G.; } \\
\text { CRUZ, D.M.C. }\end{array}$ & 2013 & $\begin{array}{l}\text { Cadernos de } \\
\text { Terapia } \\
\text { Ocupacional da } \\
\text { UFSCar }\end{array}$ \\
\hline $\begin{array}{l}\text { Eficácia de mobiliário escolar } \\
\text { adaptado de baixo custo no } \\
\text { desempenho funcional de criança } \\
\text { com paralisia cerebral }\end{array}$ & $\begin{array}{l}\text { PIOVEZANNI, } \\
\text { M.A.T.; } \\
\text { ROCHA, A.N.D.C.; } \\
\text { BRACCIALLI, }\end{array}$ & 2014 & $\begin{array}{l}\text { Revista } \\
\text { educação } \\
\text { especial }\end{array}$ \\
\hline
\end{tabular}




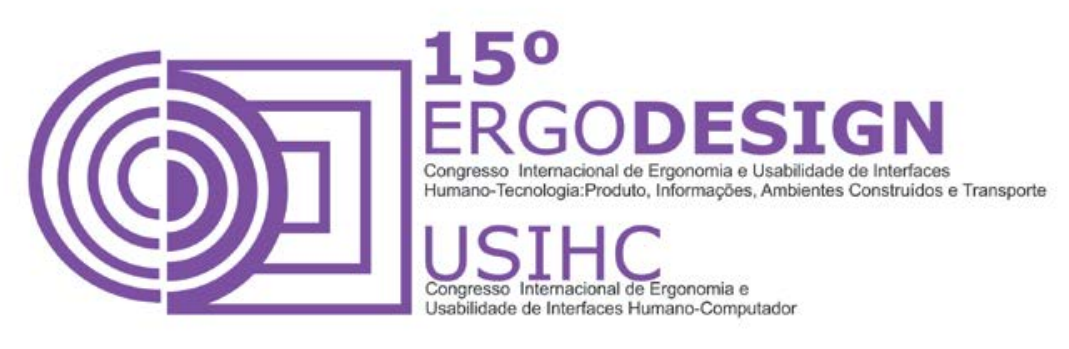

L.M.P.

O estudo de Wu et al (2003) apresenta um projeto de dispositivo assistivo para o desenho, com metodologia USERfit para ajudar crianças com paralisia cerebral com deficiência de membros superiores a superar as limitações da deficiência física em sua pintura. Com isso, para Wu et al (2003), essas crianças podem melhorar sua qualidade de vida, pois expressam sua criatividade e aliviam a tensão. Os nove elementos do USERfit foram analisados e guiaram os objetivos do projeto. São eles: contexto ambiental, ambiente do produto, análise do usuário, atividades do usuário, análise do produto, atributo do modelo do produto, resumo do requisito, resumo do design (projeto), avaliação de usabilidade.

O método utilizado na pesquisa foi um estudo de caso, tendo em vista, para Wu et al (2003), a condição complexa de múltiplas desvantagens da paralisia cerebral e as grandes diferenças de sintomas entre pacientes individuais. O caso foi uma criança com sequela de PC com deficiência motora associada a sintomas graves, incluindo fraqueza geral, incapacidade de andar, dificuldade na fala, força insuficiente para segurar canetas e incapacidade de escrever, mas com capacidade intelectual normal.

Conforme os autores supracitados, as medidas antropométricas da mão devem ser consideradas no projeto de dispositivos de assistência. Os autores realizaram as medições com um paquímetro Vernier. As medidas tomadas foram comprimento e largura da mão, comprimento da palma e comprimento da primeira articulação do dedo do meio.

O comportamento manual foi referido como parâmetro que deve ser observado, visto que, a força de preensão diminui com a flexão de punho, conforme Terrel e Purswell (1976 apud WU et al, 2003). Portanto, é essencial que as ferramentas sejam projetadas de forma a manter punhos alinhados e evitar desvios ulnar e radial, flexão palmar e dorsal (WU et al, 2003). Assim, o método de preensão da caneta influencia significativamente desempenho da escrita para a criança com PC.

A concepção do produto final, a partir da análise realizada, gerou a concepção do produto final, que incorpora três partes: (1) Dispositivo assistivo luva de preensão manual, (2) dispositivo assistivo de suporte do braço e do punho e (3) prancheta que se move (WU ET AL, 2003).

Para facilitar o uso e evitar o desvio ulnar e flexão palmar, o dispositivo apresentado inclui uma luva contendo um disco almofada em forma de $U$ para o punho evitando desvio ulnar durante o desenho. O dispositivo de suporte do braço de apoio universal, que pode ser ajustado horizontalmente e verticalmente para corresponder às necessidades do usuário e altura da mesa, foi projetado para ajudar o sujeito a superar a fraqueza do braço e da deficiência. As funções do dispositivo de suporte de apoio do braço incluem: (1) aumento da atividade do braço, (2) aumento da estabilidade do braço, e (3) o aumento da gama de desenho (WU ET AL, 2003).

Para aumentar a integridade do produto, uma prancha de desenho em movimento, com um botão de punho foi projetado para ajudar a mão esquerda para fazer avançar o papel enquanto desenha. Pérolas dos Rolling foram inseridos abaixo da prancheta de desenho, permitindo que ele seja facilmente posicionado como desejado usando o botão de punho. 


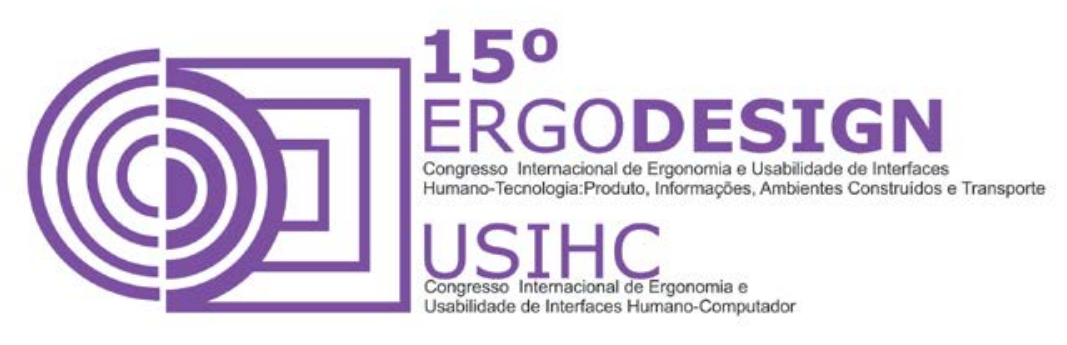

Notavelmente, esta mesa de desenho pode ser usada de acordo com a localização e capacidade do usuário. A prancheta de desenho em movimento é projetada para ajudar o usuário para mover o papel enquanto desenha, incentivar o movimento da mão esquerda, e aumentar a gama de desenho (WU ET AL, 2003).

Após avaliação deste produto final o artigo ainda indica alguns ajustes.

Através da análise de tarefas, design de produto e experiência, o estudo de Wu et al (2003) atingiu três descobertas concretas em relação à concepção de dispositivos de desenho de assistência para crianças com paralisia cerebral com deficiência de membros superiores:

5.1. Projeto de dispositivos assistivos de desenho devem começar a partir da análise dos movimentos de desenho

\subsection{Apoio de braço previne a fadiga enquanto desenha}

\subsection{Melhorar a gama de desenho e método de expressão}

Wu et al (2003) concluem que os dispositivos assistivos de desenho a partir da perspectiva de design ergonômico vinha, naquele período, recebendo menos atenção. A partir dos experimentos, o projeto do dispositivo assistivo de desenho foi eficaz em termos de movimento e alcance do desenho. Devido à variação nas capacidades comportamentais entre crianças com paralisia cerebral, este projeto dispositivo assisitivo de desenho realizado pelo método de estudo de caso pode não ser aplicável a todas as crianças com paralisia cerebral. No entanto, este projeto pode ser útil para as crianças com o mesmo tipo de deficiência ao longo das extremidades superiores e que necessitam de dispositivos de assistência para escrever ou desenhar.

Conforme Nakao, Sakamoto e Yano (2013), pessoas com deficiência física desfrutam de atividades criativas como um meio de auto expressão e motivação para a vida, e dentre estas atividades encontram-se a pintura e o desenho. Porém, para os autores supracitados, 0 ato de pintar pode ser satisfatoriamente desfrutado dependendo, em parte, do grau de deficiência do indivíduo. Movimentos involuntários afetam o controle do corpo, tornando difícil a realização de movimentos motores finos precisos, limitando assim o desempenho de tarefas desejadas, tais como pintar e desenhar.

Segundo os autores acima, para abordar estes problemas de movimentação involuntária, que desenham usando computação gráfica, estudos têm sido conduzidos em sistemas que filtram os efeitos de movimentos involuntários do sinal de entrada de um dispositivo para que o ponteiro na tela não mostre qualquer movimento involuntário. Alguns estudos têm sido desenvolvidos utilizando o método da média de movimentos para movimentos involuntários não periódicos, tais como aqueles vistos na atetose.

O grupo de pesquisa de Nakao, Sakamoto e Yano (2013) tem desenvolvido uma pesquisa, com sistema digital, voltada para pessoa com movimentos involuntários graves e para aqueles que têm dificuldade para manter o dispositivo de entrada no mesmo plano, pois a irregularidade e variabilidade da força de seus movimentos involuntários devem ser considerados. Assim, o grupo de pesquisa propôs, em pesquisa anterior, um método que altera atenuação baseado na força dos movimentos involuntários por usar a magnitude de velocidade de um dispositivo de entrada tridimensional (AOYAMA et al, 2011).

Os movimentos involuntários da atetose não podem ser previstos, e são irregulares na direção e incluem torção e reflexo. Além disso, não são apenas movimentos involuntários 


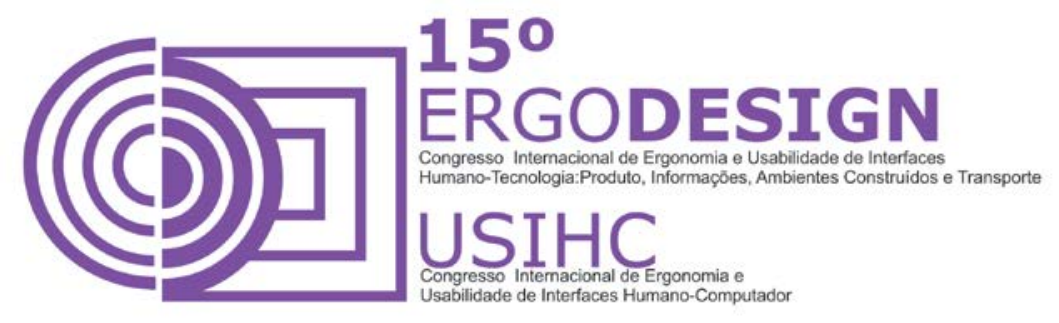

centralizados no punho, cotovelo e ombro, mas também desestabilizadores do tronco. Assim, os movimentos involuntários do atetoide são tridimensionais, movimentos complexos (AOYAMA et al, 2011).

O sistema de assistência de desenho apresentado na pesquisa de Nakao, Sakamoto e Yano (2013) consiste de um dispositivo de entrada que o utilizador manipula diretamente, um dispositivo de controlo que executa o programa, e um dispositivo de saída, que exibe os resultados do controlo. No presente estudo, também usou uma interface háptica compacto, o Phantom Omni (SensAble, Inc., Wilmington, MA).

$\mathrm{Na}$ análise do uso do sistema, Nakao, Sakamoto e Yano (2013) consideraram que os usuários com movimentos involuntários têm dificuldade para manter o dispositivo no plano e manter a preensão do dispositivo, facilidade de re-agarramento, e possibilidade de exercer força de resistência no processo de seleção de dispositivo de entrada.

Conforme Nakao, Sakamoto e Yano (2013), os movimentos involuntários de atetose incluem ambos os movimentos fracos e fortes. No caso de movimentos involuntários fortes, o usuário perde quase completamente o controle de seu movimento voluntário, também.

Nakao, Sakamoto e Yano (2013), ao analisar o desempenho dos usuários atetoides, percebeu uma série de desenhos errôneos, assim definidos quando o início do desenho e/ou o final do desenho foram executados involuntariamente.

Foi projetado então, pela equipe, o controle de correção de posição do filtro de atenuação do comportamento involuntário (AIBAF). Este tanto impede o ponteiro de invadir o alvo, mesmo quando o usuário perdeu o controle motor devido a um movimento involuntário e atenua o efeito de movimentos involuntários com mudanças na velocidade sintetizados (NAKAO; SAKAMOTO; YANO, 2013).

A maior parte dos desenhos errôneos foram causados por movimentos involuntários e isto pode levar a alongamento da criação, fadiga, tensão e significativos movimentos involuntários. Por isso, a necessidade da prevenção e redução destes erros. Usuários com movimentos involuntários muitas vezes intencionalmente desfazem desenhos errôneos. Assim, para os autores supracitados, deve-se pensar também no dispositivo ou estratégias para apagar o desenho, que no caso da pesquisa era por meio de um botão de desfazer na tela do computador.

Nos testes de usuário, observou-se que as causas de desenhos errôneos compõem quatro categorias básicas: segurando (preensão) a caneta, movendo o ponteiro, fraqueza e expressão não intencional de um ponto no início do desenho/expressão de uma linha (NAKAO; SAKAMOTO; YANO, 2013). Os erros atribuídos têm como causa suspeita um desequilíbrio entre os músculos agonistas, sinérgicos e antagonistas, como as características dos movimentos involuntários.

Nakao, Sakamoto e Yano (2013) concluíram que o sistema assistivo de desenho com um filtro de atenuação do comportamento involuntário adaptativo integrado muda a atenuação aos movimentos involuntários dos usuários e manipulação em tempo real, além de prevenir o erro no desenho com um controlador de força de resistência.

O estudo de Plotegher, Emmel e Cruz (2013) teve como objetivo relatar a utilização dos produtos assistivos como auxílio no desempenho escolar de alunos com deficiência incluídos na rede regular de ensino de São Carlos, no estado de São Paulo. Crianças com 


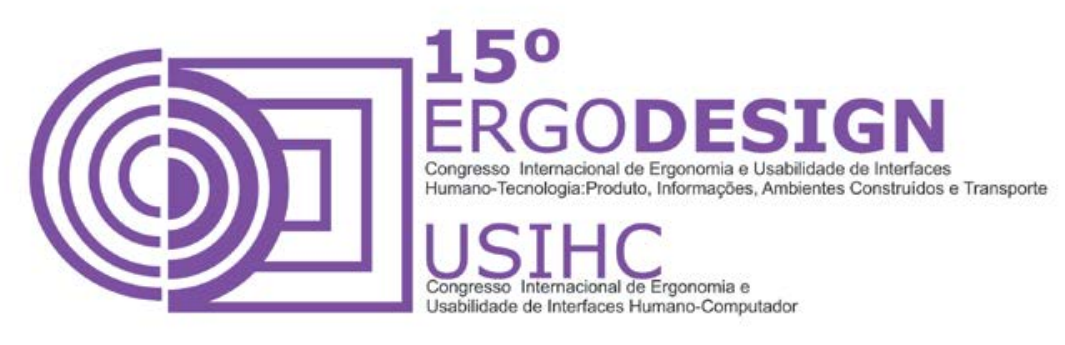

deficiência física foram avaliadas por meio da aplicação da avaliação SFA (School Function Assessment) com as professoras dos alunos e, posteriormente, a observação do desempenho destes alunos em sala de aula, mais especificamente de sua função manual.

A SFA aborda o perfil funcional do aluno e informa sobre a participação ativa dele em atividades e tarefas da rotina escolar, sem incluir o desempenho no conteúdo acadêmico. Foram avaliadas as atividades que poderiam estar relacionadas, tais como: manter o papel sobre a mesa, utilizar borracha, régua, preensão do lápis ou caneta, uso do caderno, recortar etc. Todos os alunos que apresentaram dificuldades na SFA receberam um registro detalhando tais problemas e esse registro guiou a tomada de decisão acerca de suas necessidades com relação a produtos de tecnologia assistiva.

Conforme Plotegher, Emmel e Cruz (2013), após este levantamento, das dificuldades e necessidades do aluno, foram estudados quais tipos de adaptações seriam necessários para cada caso, porém restritos a produtos de baixo custo.

Dos 13 alunos estudados por Plotegher, Emmel e Cruz (2013), a maioria possuía o diagnóstico de paralisia cerebral (PC). Dentre as maiores dificuldades observadas, estava o uso de materiais para escrita e desenho (como lápis, pincel, caneta, apontador, borracha, clipes, grampo).

Quanto aos dispositivos indicados e confeccionados com maior frequência relacionados às atividades de escrita e desenho, foram citados: adaptações para lápis, giz de cera, caneta e cola (normalmente engrossadores); adaptações nos apontadores de lápis; plano inclinado; fixadores de folhas e cadernos para mesa; janela retangular para escrita e leitura. Os engrossadores de lápis foram confeccionados com EVA (PLOTEGHER; EMMEL; CRUZ, 2013).

O artigo acima citado não descreve as dificuldades apresentadas por cada criança especificamente, nem o processo de escolha dos artefatos e nem avaliação da eficácia dos mesmos na função escolar, de modo que não apresenta detalhadamente parâmetros e requisitos considerados para o desenvolvimento dos artefatos.

O estudo de Piovezanni, Rocha e Braccialli (2014) teve como objetivo confeccionar uma cadeira e mesa escolar adaptadas com material de baixo custo e verificar sua eficácia no desempenho grafomotor de uma criança com paralisia cerebral diplégica. A coleta e registro dos dados foram feitos em três momentos, com a criança posicionada no mobiliário adaptado, no mobiliário escolar comum e, novamente, no mobiliário adaptado, para se estabelecer a comparação.

Para a coleta e análise dos dados, foram utilizados na pesquisa acima citada: a cadeira adaptada e mesa recortada confeccionadas na pesquisa, mesa e cadeira escolar comum, computador e mesa digitalizadora da marca Wacom - Intuos 3. Para análise da produção gráfica do sujeito da pesquisa foi usado o software MovAlyseR 6.1., por meio das variáveis tempo de duração da atividade, velocidade (capacidade de deslocar a caneta no espaçotempo), aceleração (capacidade do indivíduo para alterar sua aceleração durante o movimento) e pressão da caneta no papel. Para a análise dos dados de tempo de duração da atividade, velocidade, aceleração e pressão da caneta foi feito o teste de normalidade de Shapiro Wilk (PIOVEZANNI; ROCHA; BRACCIALLI, 2014).

Neste estudo acima referido foi realizada uma comparação do desempenho grafomotor da criança com os 3 mobiliários diferentes, porém, pela análise de significância, não se pôde 


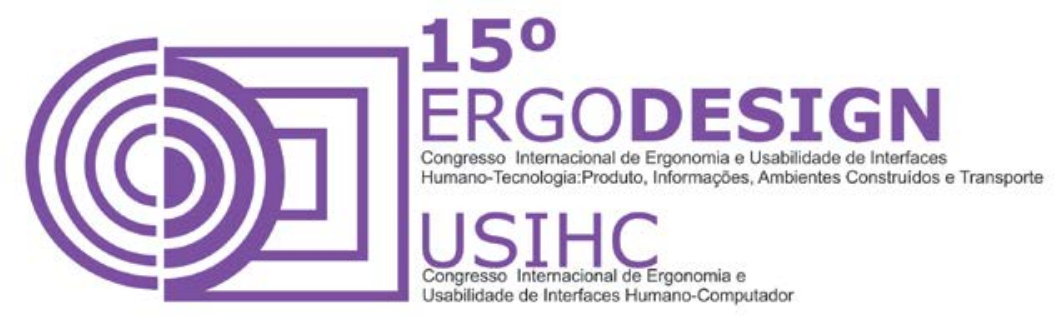

afirmar que o mobiliário adaptado confeccionado especificamente para o participante do estudo interferiu no desempenho grafomotor. Vale ressaltar que a criança do estudo era diplégica, que é um dos tipos de PC mais leves, as quais apresentam menor comprometimento de membros superiores.

Roon, Steenbergen, Meulenbroek (2005) examinaram, em sua pesquisa, se ser capaz de monitorar visualmente o movimento dos membros é uma pré-condição para as pessoas com PC fazerem movimentos precisos de membro superior. Oito participantes com PC tetraparéticos e oito controles sem histórico de disfunção neurológica foram convidados a produzir em larga amplitude, desenhando movimentos em uma mesa digitalizadora em linha reta.

A precisão de movimento quando a visão foi bloqueada foi comparável nos dois grupos. Os autores concluíram que as pessoas com PC tetraparéticos não exigem estritamente visão constante de seu membro em movimento para fazer movimentos precisos de membro superior, mas eles compensam, pela falta de informação visual, com um prolongamento do tempo de movimento. A força desempenhada no uso da caneta provou ser uma adaptação estratégica geral, possivelmente para filtrar o ruído indesejado do sistema motor ou para melhorar a entrada proprioceptiva.

Em revisão de literatura, Graham e Weintraub (1996) vêm a confirmar esta informação acima, de que recentes pesquisas demonstraram que visão é importante, mas não absolutamente necessário ingrediente no controle da escrita.

Porém, é importante pontuar que, conforme conceitos de Jordan (1998), pelo que o artigo acima aponta a precisão não foi perdida, o que poderia se considerar como eficácia por se conseguir completar a tarefa, porém, não se atingiu eficiência, pois pelo comprometimento da visão exigiu-se maior esforço e tempo.

A seguir (tabela 2) são explicitados os parâmetros e requisitos encontrados nos artigos.

Tabela 2

\begin{tabular}{|c|l|}
\hline \multicolumn{1}{|c|}{$\begin{array}{c}\text { Parâmetros } \\
\text { (o que devo considerar para projetar o } \\
\text { artefato?) }\end{array}$} & \multicolumn{1}{|c|}{$\begin{array}{c}\text { Requisitos } \\
\text { (o que pretendo proporcionar com o } \\
\text { artefato?) }\end{array}$} \\
\hline $\begin{array}{l}\text { contexto ambiental, ambiente do produto, } \\
\text { análise do usuário, atividades do usuário, } \\
\text { análise do produto, atributo do modelo do } \\
\text { produto, resumo do requisito, resumo do } \\
\text { design (projeto), avaliação de usabilidade, } \\
\text { análise dos movimentos de desenho }\end{array}$ & $\begin{array}{l}\text { manter punhos alinhados e evitar } \\
\text { desvios ulnar e radial, flexão palmar e }\end{array}$ \\
\hline medidas antropométricas da mão & $\begin{array}{l}\text { aumento da atividade do braço; } \\
\text { aumento da estabilidade do braço e } \\
\text { apoio do braço; e o aumento da gama de } \\
\text { desenho }\end{array}$ \\
\hline
\end{tabular}




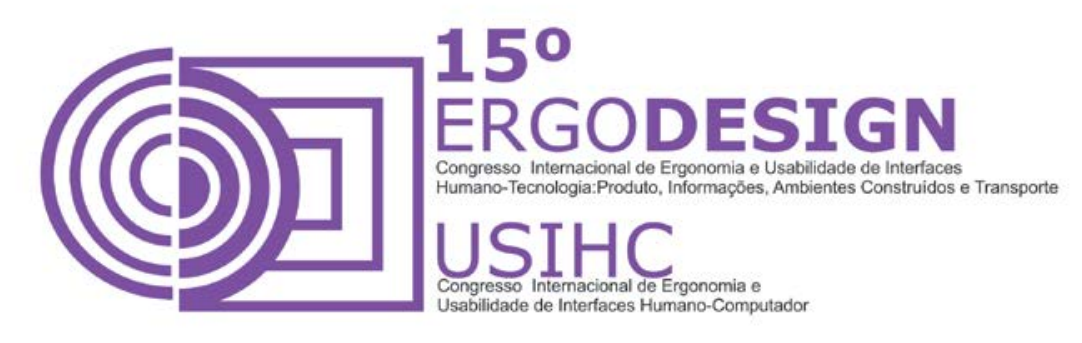

\begin{tabular}{|l|l|}
\hline comportamento manual / função manual & $\begin{array}{l}\text { manter o dispositivo/ artefato no plano; } \\
\text { manter a preensão do dispositivo; } \\
\text { facilidade de re-agarramento }\end{array}$ \\
\cline { 1 - 2 } grau de deficiência do indivíduo & possibilidade de apagar o desenho \\
\cline { 1 - 2 } $\begin{array}{l}\text { irregularidade e variabilidade da força dos } \\
\text { movimentos involuntários }\end{array}$ & \multirow{2}{*}{$\begin{array}{l}\text { usabilidade de materiais para escrita e de movimento } \\
\text { desenho }\end{array}$} \\
\cline { 1 - 2 } $\begin{array}{l}\text { tempo de duração da atividade de desenho, } \\
\text { velocidade, aceleração e pressão da caneta }\end{array}$ & \\
\cline { 1 - 2 } visão & \\
\hline
\end{tabular}

\section{CONSIDERAÇÕES FINAIS}

Por meio da pesquisa realizada foi possível identificar alguns parâmetros e requisitos apresentados nos artigos relacionados ao desempenho da criança com sequelas de paralisia cerebral. Alguns parâmetros citados não foram criados pelos autores, mas já compõem metodologias identificadas nos artigos. A literatura aqui apresentada foi apenas 0 início deste levantamento, mas sabe-se que muitos artigos que abordam artefatos para este fim não detalham os parâmetros e/ou requisitos considerados no projeto dos mesmos. Porém, entende-se a importância de se estabelecer estes critérios para a contribuição dos projetos, pois, a usabilidade e funcionalidade de cada criança com sequela de paralisia cerebral vão ser muito diversificadas.

\section{REFERÊNCIAS BIBLIOGRÁFICAS}

ASSOCIAÇÃO BRASILEIRA DE NORMAS TÉCNICAS. NBR 9241-11: requisitos ergonômicos para trabalho de escritórios com computadores: parte 11- orientações sobre usabilidade. Rio de Janeiro, 2002.

AOYAMA, $\mathrm{H}$. et al. Development of drawing assist system for patients with cerebral palsy of the tension athetosis type. IEEE International Conference on Robotics and Automation, p. 46644669, maio 2011.

CASSIS;, L.; FRANCISQUETTI., A. A. Avaliaçao da Imagem em crianças portadoras de paralisia cerebral através da história de "Alice no pais das maravilhas." arquivos brasileiros de paralisia cerebral, v. 15, n. 89/90, 2006.

CAVATON, M.F.F.; BARBATO, S. B. A mediação da fala, do desenho e da escrita na construção de conhecimento da criança de seis anos. [s.l.] Universidade de Brasília, 2010. 


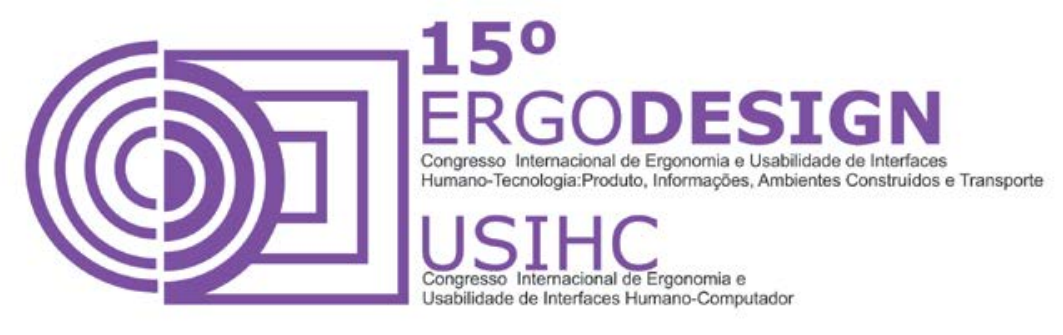

CAZEIRO, A. P. M.; LOMÔNACO, J. F. B. Formação de conceitos por crianças com paralisia cerebral: um estudo exploratório sobre a influência de atividades lúdicas. Psicologia: reflexão e crítica, v. 24, n. 1, p. 40-50, 2011.

DICIO: Dicionário Online de Português, definições e significados de mais de 400 mil palavras. Disponível em: <http://www.dicio.com.br/parametro/>. Acesso em: 11 fevereiro 2015.

GAUZZI, L.D.V.; FONSECA, L.F. Classificação da Paralisia Cerebral. In: LIMA, C.L.F.A.; FONSECA, L.F. (Org.). Paralisia Cerebral: neurologia, ortopedia, reabilitação. Rio de Janeiro: Guanabara Koogan, 2004. p. 37-44.

GRAHAM, S.; WEINTRAUB, N. A review of handwriting research: Progress and prospects from 1980 to 1994. Educational Psychology Review, v. 8, n. 1, p. 7-87, mar. 1996.

JORDAN, Patrick W. An introduction to usability. London: Taylor \& Francis, 1998.

KAVAK, S. T.; BUMIN, G. The effects of pencil grip posture and different desk designs on handwriting performance in children with hemiplegic cerebral palsy. Jornal de Pediatria, v. 85, n. 4, p. 346-352, 7 ago. 2009.

KRIGGER, K. W. Cerebral Palsy: An Overview. American Family Physician, v. 73, n. 1, 2006.

NAKAO, T.; SAKAMOTO, R.; YANO, K. Drawing Assist System Considering Nonperiodic Involuntary Movements. 12 th IFAC Sumposium on Analysis, Designand Evaluation of Human-Machine Systems, n. 2005, 2013.

PIOVEZANNI, M. A. T.; ROCHA, A. N. D. C.; BRACCIALLI, L. M. P. Eficácia de mobiliário escolar adaptado de baixo custo no desempenho funcional de criança com paralisia cerebral. Revista educaçao especial, v. 27, n. 49, p. 485-498, 2014.

PLOTEGHER, C. B.; EMMEL, M. L. G.; CRUZ, D. M. C. DA. Utilização de dispositivos assistivos por alunos com deficiência em escolas públicas. Cadernos de Terapia Ocupacional da UFSCar, v. 21, n. 1, p. 35-42, 2013.

ROON, D. V.; STEENBERGEN, B.; MEULENBROEK, R. G. J. Movement-Accuracy Control in Tetraparetic Cerebral Palsy: Effects of Removing Visual Information of the Moving Limb. Motor Control, p. 372-394, 2005.

SHUMWAY-COOK, A.; WOOLLACOTT, M.H. Controle motor: teoria e aplicações práticas. São Paulo: Manole, 2003.

SILVA, N. M. V.; VASCONCELOS, A. N. O self dialogico no desenho infantil. Psicologia: reflexão e crítica, v. 26, n. 2, p. 346-356, 2013.

WU, F. et al. Assistive drawing device design for Cerebral Palsy children. Technology and Disability, v. 15, p. 239-246, 2003. 